\title{
AVALIAÇÃO DO VIGOR DE SEMENTES DE RÚCULA PELO TESTE DE LIXIVIAÇÃO DE POTÁSSIO ${ }^{1}$
}

\author{
CHARLINE ZARATIN ALVES², MARCO EUSTÁQUIO DE SÁ ${ }^{3}$
}

\begin{abstract}
RESUMO - Objetivou-se neste trabalho estudar metodologias para a condução do teste de lixiviação de potássio, verificando sua eficiência na identificação de diferentes níveis de vigor de lotes de sementes de rúcula. Para tanto, o estudo foi conduzido utilizando cinco lotes de sementes de Rúcula Cultivada e cinco lotes de sementes de Rúcula Gigante. Foram realizados os testes de germinação, primeira contagem de germinação, emergência de plântulas em casa de vegetação e variações no teste de lixiviação de potássio (temperaturas de $25^{\circ} \mathrm{C}$ e $30^{\circ} \mathrm{C}$; volumes de $50 \mathrm{~mL}$ e $75 \mathrm{~mL}$ de água; 50 e 100 sementes; e períodos de 0,5 h, 1 h, 1,5 h, 2 h, 2,5 h, 3, 4 e 5 horas). Diante dos resultados obtidos, verificou-se que o período de embebição para o teste de lixiviação de potássio pode ser reduzido para 2 horas e o volume de água utilizado pode ser reduzido para 50 $\mathrm{mL}$. As combinações de 50 sementes apresentaram menor coeficiente de variação e a temperatura mais adequada foi a de $30^{\circ} \mathrm{C}$. Assim, concluiu-se que a condição mais adequada para o teste de lixiviação de potássio em sementes de rúcula é a utilização de 50 sementes em $50 \mathrm{~mL}$ de água por 2 horas, a $30^{\circ} \mathrm{C}$.
\end{abstract}

Termos para indexação: Eruca sativa, sementes, potencial fisiológico.

\section{POTASSIUM LEACHATE IN ARUGULA SEEDS EVALUATION OF ARUGULA SEED VIGOR BY THE POTASSIUM LEACHING TEST}

\begin{abstract}
The objective of this study was to evaluate methodologies for the potassium leaching test and to verify its sensitivity for identifying different levels of vigor of arugula seed lots. Five seed lots each of Rucula Cultivada and Rucula Gigante were used. Standard germination, first count of germination, seedling emergence and variations of the potassium leaching test (50 or 100 seeds imbibed in $50 \mathrm{~mL}$ or $75 \mathrm{~mL}$ in water; at $25^{\circ} \mathrm{C}$ and $30^{\circ} \mathrm{C}$; for $0.5 \mathrm{~h}, 1 \mathrm{~h}, 1.5 \mathrm{~h}, 2 \mathrm{~h}$, $2.5 \mathrm{~h}, 3,4$ and 5 hours) were done. It was observed that the period seeds need to be soaked can be reduced to 2 hours and the water volume can be reduced to $50 \mathrm{~mL}$. The utilization of 50 seeds showed a smaller variation coefficient and the best temperature was $30{ }^{\circ} \mathrm{C}$. It can be concluded that the potassium leaching test for arugula seeds can be run using 50 seeds in $50 \mathrm{~mL}$ of water for 2 hours at $30^{\circ} \mathrm{C}$.
\end{abstract}

Index terms: Eruca sativa, seeds, physiological potential.

\footnotetext{
${ }^{1}$ Submetido em 30/09/2009. Aceito para publicação em 06/01/2010.

${ }^{2}$ Professora Adjunta do Câmpus de Chapadão do Sul - Universidade Federal de Mato Grosso do Sul/UFMS - Acesso à Fazenda Campo Bom pela Rodovia MS-306, km 105, C.P: 112 - 79560-000 - Chapadão do Sul/ MS-charline@ufms.br.
}

${ }^{3}$ Professor titular do Departamento de Fitotecnia, Tecnologia de Alimentos e Sócio-Economia - UNESP, Av. Brasil n 56, C.P. 31, CEP 15385-000, Ilha Solteira/SP - mesa@agr.feis.unesp.br. 


\section{INTRODUÇ̃̃O}

A hortaliça folhosa mais plantada e consumida pela população brasileira é a alface, porém desde o final da década de 90, a rúcula (Eruca sativa) vem conquistando maior espaço no mercado, onde houve aumento na quantidade comercializada de $78 \%$, de 1997 para 2003. Esse crescimento pode ser considerado significativo, quando comparado com o da alface americana e crespa, que apresentou um aumento de $40 \%$ nesse mesmo período (Purquerio, 2005).

A qualidade da semente utilizada no processo de produção agrícola é um dos principais fatores a ser considerado para a implantação da cultura e há consenso entre os pesquisadores, tecnologistas e produtores de sementes sobre a importância do vigor das sementes e da necessidade de avaliá-lo. Resultados de pesquisa mostram que a baixa qualidade fisiológica de sementes pode resultar em reduções na velocidade e emergência total, desuniformidade de emergência, menor tamanho inicial de plântulas, produção de matéria seca e na área foliar (Höfs et al., 2004, Kolchinski et al., 2006).

Estudado com menor frequência, o teste de lixiviação de potássio baseia-se no mesmo princípio da condutividade elétrica, variando apenas o parâmetro avaliado e se constitui em alternativa para avaliar o vigor de sementes. Esse teste apresenta a vantagem de avaliar um íon específico, permitindo obter informações sobre o potencial fisiológico dos lotes com maior rapidez e agilidade (Dias e Marcos Filho, 1995).

O principal íon lixiviado pelas sementes é o potássio (Lott et al, 1991). Em sementes de ervilha e de feijão, Mullett e Considine (1980) verificaram que 25 a $50 \%$ do total de eletrólitos liberados correspondem ao íon $\mathrm{K}^{+}$. Resultados semelhantes foram obtidos por Woodstock et al. (1985) em sementes de algodão.

Dessa forma, a quantidade de potássio liberada por sementes embebidas tem sido utilizada como indicador da integridade do sistema de membranas celulares (Halloin, 1975, McKersie e Stinson, 1980, Woodstock et al, 1985). Em sementes de ervilha, Simon e Raja-Harun (1972) observaram estreita relação entre os resultados de condutividade elétrica e quantidade de potássio lixiviado após 24 horas de embebição. Halloin (1975) verificou que, em sementes de algodão, a concentração de íons potássio lixiviados após 60 minutos de embebição correspondeu à condutividade elétrica avaliada com 15 e 45 minutos. Moss e Mullet (1982), em sementes de feijão, verificaram que a quantidade de potássio lixiviado após 72 horas pode ser considerado como parâmetro indicativo de vigor. Marcos Filho et al (1982) observaram que a lixiviação de potássio decresceu proporcionalmente com a elevação do poder germinativo e vigor de sementes de soja.
A rapidez do teste foi observada por Custodio e Marcos Filho (1997), que verificaram diferenças de vigor em sementes de soja após 30 minutos de embebição. Porém, Torres (2002) concluiu que o teste de lixiviação de potássio em sementes de melão, necessita de estudos adicionais para adequar sua metodologia, considerando inclusive, os prováveis efeitos do genótipo.

A liberação de potássio está diretamente ligada ao estado das membranas e independe da quantidade de potássio nas sementes, conforme demonstraram Panobianco e Marcos Filho (2001), em sementes de tomate; e Miguel (2001), em sementes de milho.

Em outras hortaliças, Simon e Mathavan (1986) relataram que espécies como aipo, alface e cenoura apresentaram $90 \%$ da lixiviação de potássio num período de 5 a 15 minutos de embebição, tornando-o um teste ainda mais promissor em relação à rapidez das informações obtidas. No entanto, para sementes de rúcula, não há na literatura informações sobre metodologia específica para condução deste teste, justificando, portanto, a necessidade de intensificação da pesquisa para esta espécie.

\section{MATERIAL E MÉTODOS}

O trabalho foi conduzido no Laboratório de Produção e Tecnologia de Sementes da Universidade Estadual Paulista "Júlio de Mesquita Filho" - UNESP - do Câmpus de Ilha Solteira/SP, no período de fevereiro a setembro de 2007, utilizando-se cinco lotes de sementes de dois cultivares de rúcula. Foram determinados: o grau de umidade, porcentagem de germinação, primeira contagem de germinação, emergência de plântulas, e procedimentos específicos para a condução do teste de lixiviação de potássio.

As sementes recebidas estavam embaladas em recipientes herméticos do tipo lata, e durante todo o período experimental, as mesmas permaneceram armazenadas em câmara seca a $20^{\circ} \mathrm{C}$ e $50 \%$ de umidade relativa do ar.

Determinação do grau de umidade: foi realizada através do método da estufa, durante 24 horas, a 105+/$3{ }^{\circ} \mathrm{C}$, de acordo com as Regras para Análise de Sementes (Brasil, 1992). Foram utilizadas duas subamostras com aproximadamente, $2,0 \mathrm{~g}$ de sementes para cada lote.

Teste de Germinação: foram utilizadas quatro subamostras de 50 sementes por lote, distribuídas sobre uma folha de papel germitest, previamente umedecidas com quantidade de água equivalente a 2,5 vezes a massa do substrato, colocadas no interior de caixas plásticas transparentes $(11,5 \times 11,5 \times 3,5 \mathrm{~cm})$, sendo mantidas em germinador a $25{ }^{\circ} \mathrm{C}$. As avaliações foram realizadas aos quatro e sete dias após a semeadura, segundo os 
critérios estabelecidos pelas Regras para Análise de Sementes (Brasil, 1992).

Primeira Contagem de Germinação: foi realizada juntamente com o teste de germinação, computando-se a porcentagem média de plântulas normais, obtidas aos quatro dias após a semeadura.

Emergência de plântulas: foram utilizadas bandejas de isopor com 128 células individuais, contendo substrato comercial Plantmax Hortaliças. As bandejas foram mantidas em casa de vegetação com controle de temperatura $\left(25^{\circ} \mathrm{C}\right)$, com irrigações diárias (3 vezes ao dia). Foram utilizadas quatro subamostras de 50 sementes por lote, colocando-se uma semente por célula. A avaliação da emergência das plântulas foi efetuada aos 10 dias após a semeadura, mediante a contagem de plântulas normais emergidas, avaliadas de acordo com os critérios adotados para avaliação da parte aérea de plântulas normais em um teste de germinação.

Lixiviação de potássio: foram estudados os efeitos do período de embebição $(0,5 \mathrm{~h}, 1 \mathrm{~h}, 1,5 \mathrm{~h}, 2 \mathrm{~h}, 2,5 \mathrm{~h}$, 3 , 4 e $5 \mathrm{~h}$ ), do volume de água destilada $(50$ e $75 \mathrm{~mL})$, do número de sementes $(50$ e 100$)$ e da temperatura de embebição $\left(25^{\circ} \mathrm{C}\right.$ e $\left.30^{\circ} \mathrm{C}\right)$.

$\mathrm{O}$ teste foi conduzido utilizando-se quatro subamostras de 50 ou 100 sementes previamente pesadas (precisão de 0,0001 g), colocadas em copos plásticos contendo água destilada e mantidas em germinador durante cada período e temperaturas previstas para a embebição. As leituras foram efetuadas em fotômetro de chama ANALYSER - 910, ressaltando a facilidade de leitura deste aparelho.

Para o cálculo da lixiviação de potássio considerouse a leitura obtida no fotômetro de chama, o volume de água destilada utilizado $(\mathrm{mL})$ e a massa da amostra $(\mathrm{g})$. $\mathrm{O}$ resultado final foi expresso em $\mu \mathrm{g}$ de $\mathrm{K} / \mathrm{g}$ de semente, ou seja, ppm de potássio.

Os dados referentes aos testes de germinação, primeira contagem de germinação e emergência de plântulas em casa de vegetação foram transformados em arc sen $\sqrt{x}_{\mathrm{X}} / 100$, enquanto que os dados de lixiviação de potássio não sofreram transformação.

As análises de variância foram realizadas separadamente para cada cultivar e teste conduzido, utilizando-se o delineamento inteiramente casualizado, com quatro repetições, sendo que a comparação múltipla das médias foi realizada através do Teste de Tukey, no nível de $5 \%$ de probabilidade, utilizando o programa SANEST - Sistema de Análise Estatística (Zonta e Machado, 1984). Determinou-se também o coeficiente de correlação simples entre os resultados dos testes de avaliação da qualidade fisiológica das sementes e o teste de emergência em casa de vegetação.

\section{RESULTADOS E DISCUSSÃO}

A avaliação inicial da qualidade fisiológica dos lotes de sementes de rúcula foi efetuada pelos testes de germinação, primeira contagem de germinação e emergência de plântulas em casa de vegetação. Nessa etapa, também foi avaliado o grau de umidade das sementes. Os resultados dessas avaliações se encontram na Tabela 1 .

\section{TABELA 1. Qualidade inicial de cinco lotes dos cultivares Rúcula Cultivada e Rúcula Gigante.}

\begin{tabular}{lccccc}
\hline Cultivares & Lotes & $\begin{array}{c}\text { Grau de } \\
\text { Umidade }\end{array}$ & Germinação & $\begin{array}{c}\text { Primeira } \\
\text { Contagem }\end{array}$ & $\begin{array}{c}\text { Emergência } \\
\text { de Plântulas }\end{array}$ \\
\hline & 1 & 5,5 & $98 \mathrm{a}$ & $96 \mathrm{a}$ & $98 \mathrm{a}$ \\
& 2 & 5,0 & $93 \mathrm{a}$ & $82 \mathrm{~b}$ & $89 \mathrm{~b}$ \\
Rúcula & 3 & 5,4 & $97 \mathrm{a}$ & $94 \mathrm{a}$ & $96 \mathrm{a}$ \\
Cultivada & 4 & 5,5 & $85 \mathrm{~b}$ & $73 \mathrm{c}$ & $78 \mathrm{c}$ \\
& 5 & 5,0 & $96 \mathrm{a}$ & $95 \mathrm{a}$ & $97 \mathrm{a}$ \\
\hline & $\mathrm{CV}(\%)$ & - & 3,3 & 4,0 & 6,2 \\
\hline \multirow{6}{*}{ Rúcula } & 6 & 5,5 & $96 \mathrm{a}$ & $96 \mathrm{a}$ & $96 \mathrm{a}$ \\
Gigante & 8 & 5,9 & $98 \mathrm{a}$ & $84 \mathrm{~b}$ & $89 \mathrm{~b}$ \\
& 9 & 5,7 & $94 \mathrm{a}$ & $72 \mathrm{c}$ & $75 \mathrm{c}$ \\
& 10 & 5,3 & $97 \mathrm{a}$ & $95 \mathrm{a}$ & $98 \mathrm{a}$ \\
\hline
\end{tabular}

* Letras distintas dentro de cada coluna diferem entre si pelo Teste de Tukey, a $5 \%$ de probabilidade.

Verifica-se que para os lotes da Rúcula Cultivada, o teste de germinação destacou o lote 4 como de pior qualidade fisiológica, diferindo estatisticamente dos demais, ou seja, os lotes 1,3 e 5 foram agrupados num mesmo nível de vigor, não diferindo entre si. Os testes de primeira contagem de germinação e emergência de plântulas em casa de vegetação confirmaram o lote 4 como o de pior qualidade e permitiu destacar os lotes 1,3 e 5 como os de melhor vigor, não diferindo estatisticamente entre si. O lote 2 se mostrou como intermediário, conforme Tabela 1.

Já os resultados dos lotes da Rúcula Gigante não diferiram entre si no teste de germinação. Porém, resultados elevados e semelhantes no teste de germinação 
não significam necessariamente que todos os lotes possuem alto vigor, uma vez que o teste de germinação é conduzido em condições favoráveis de temperatura, umidade e luminosidade, permitindo ao lote a expressão do potencial máximo para produzir plântulas normais (Marcos Filho, 1999). Nesse sentido, os testes de primeira contagem de germinação e emergência de plântulas em casa de vegetação apresentaram maior sensibilidade (Tabela 1), indicando a menor qualidade fisiológica dos lotes 8 e 9 em comparação aos demais lotes.

De maneira semelhante ao teste de primeira contagem de germinação, o teste de emergência de plântulas em casa de vegetação permitiu classificar para os dois cultivares, os lotes de melhor e pior desempenho (Tabela 1). Os testes de vigor, além de ranquear os lotes, devem associarse ao desempenho das plântulas em campo, a fim de um monitoramento da eficiência dos procedimentos adotados em laboratório (Marcos Filho, 1999), o que foi conseguido nos trabalhos de Piana et al. (1995) em sementes de cebola, e Larsen et al. (1998) com sementes de nabo e ervilha.

Nas Tabelas 2 a 5 encontram-se os dados obtidos para o teste de lixiviação de potássio, envolvendo as combinações número de sementes/volume de água/ temperatura.

TABELA 2. Dados médios obtidos para lixiviação de potássio $(\mu \mathrm{g} \mathrm{K} / \mathrm{g})$, utilizando as combinações 50 sementes/50 mL e 50 sementes/75 mL, a $25{ }^{\circ} \mathrm{C}$, de cinco lotes de sementes dos cultivares Rúcula Cultivada e Rúcula Gigante.

\begin{tabular}{|c|c|c|c|c|c|c|c|c|c|}
\hline \multirow{2}{*}{ Cultivares } & \multirow{2}{*}{ Lotes } & \multicolumn{8}{|c|}{ Períodos de embebição } \\
\hline & & 0,5 & 1 & 1,5 & 2 & 2,5 & 3 & 4 & 5 \\
\hline & \multicolumn{9}{|c|}{50 sementes $/ 50 \mathrm{~mL} / 25^{\circ} \mathrm{C}$} \\
\hline \multirow{5}{*}{$\begin{array}{l}\text { Rúcula } \\
\text { Cultivada }\end{array}$} & 1 & $425 \mathrm{a}$ & $518 \mathrm{a}$ & $688 \mathrm{a}$ & $721 \mathrm{a}$ & $821 \mathrm{a}$ & $823 \mathrm{a}$ & $932 \mathrm{a}$ & $960 \mathrm{a}$ \\
\hline & 2 & $877 \mathrm{~b}$ & $1084 \mathrm{~b}$ & $1151 \mathrm{~b}$ & $1204 \mathrm{c}$ & $1267 \mathrm{c}$ & $1279 \mathrm{c}$ & $1284 \mathrm{~b}$ & $1298 \mathrm{~b}$ \\
\hline & 3 & 463 a & $537 \mathrm{a}$ & 659 a & $736 \mathrm{a}$ & $784 \mathrm{a}$ & 816 a & 905 a & $927 \mathrm{a}$ \\
\hline & 4 & $470 \mathrm{a}$ & $552 \mathrm{a}$ & $675 \mathrm{a}$ & 793 a & $870 \mathrm{~b}$ & $903 \mathrm{~b}$ & $981 \mathrm{ab}$ & $1216 b$ \\
\hline & 5 & $811 \mathrm{~b}$ & $915 \mathrm{~b}$ & $976 \mathrm{~b}$ & $1054 \mathrm{~b}$ & $1132 \mathrm{bc}$ & $1157 \mathrm{bc}$ & $1173 \mathrm{~b}$ & $1193 \mathrm{~b}$ \\
\hline & \multicolumn{9}{|c|}{ C.V. $(\%)=3,8$} \\
\hline \multirow{7}{*}{ Rúcula Gigante } & 6 & 763 a & $839 \mathrm{a}$ & $880 \mathrm{a}$ & 881 a & $893 \mathrm{a}$ & $894 \mathrm{a}$ & $905 \mathrm{a}$ & $965 \mathrm{a}$ \\
\hline & 7 & $1241 \mathrm{~b}$ & $1347 \mathrm{~b}$ & $1404 \mathrm{~b}$ & $1422 \mathrm{~b}$ & $1423 \mathrm{~b}$ & $1427 \mathrm{~b}$ & $1436 \mathrm{~b}$ & $1438 \mathrm{~b}$ \\
\hline & 8 & $1608 \mathrm{c}$ & $1676 \mathrm{c}$ & $1735 \mathrm{c}$ & $1758 \mathrm{c}$ & $1807 \mathrm{c}$ & $1812 \mathrm{c}$ & $1819 \mathrm{c}$ & $1822 \mathrm{c}$ \\
\hline & 9 & $869 \mathrm{ab}$ & $961 \mathrm{ab}$ & $1043 \mathrm{ab}$ & $1064 \mathrm{ab}$ & $1076 \mathrm{ab}$ & $1081 \mathrm{ab}$ & $1092 \mathrm{ab}$ & $1113 a b$ \\
\hline & 10 & 674 a & $764 \mathrm{a}$ & 822 a & 879 a & 881 a & 893 a & 944 a & 967 a \\
\hline & \multicolumn{9}{|c|}{ C.V. $(\%)=4,1$} \\
\hline & \multicolumn{9}{|c|}{50 sementes $/ 75 \mathrm{~mL} / 25^{\circ} \mathrm{C}$} \\
\hline \multirow{5}{*}{$\begin{array}{l}\text { Rúcula } \\
\text { Cultivada }\end{array}$} & 1 & $371 \mathrm{a}$ & $473 \mathrm{a}$ & $635 \mathrm{a}$ & $674 \mathrm{a}$ & $771 \mathrm{a}$ & $773 \mathrm{a}$ & 889 a & $915 \mathrm{a}$ \\
\hline & 2 & $822 \mathrm{~b}$ & $1038 \mathrm{c}$ & $1097 \mathrm{~b}$ & $1158 \mathrm{~b}$ & $1215 \mathrm{~b}$ & $1224 \mathrm{c}$ & $1232 \mathrm{c}$ & $1246 \mathrm{~b}$ \\
\hline & 3 & $418 \mathrm{a}$ & $487 \mathrm{a}$ & $606 \mathrm{a}$ & $686 \mathrm{a}$ & $738 \mathrm{a}$ & $768 \mathrm{a}$ & 857 a & 878 a \\
\hline & 4 & 420 a & $493 \mathrm{a}$ & $622 \mathrm{a}$ & $744 \mathrm{a}$ & 821 a & $859 \mathrm{~b}$ & $936 \mathrm{~b}$ & $1162 \mathrm{~b}$ \\
\hline & 5 & $759 \mathrm{~b}$ & $865 \mathrm{~b}$ & $920 \mathrm{~b}$ & $1009 \mathrm{~b}$ & $1080 \mathrm{~b}$ & $1103 b c$ & $1120 b c$ & $1131 \mathrm{~b}$ \\
\hline & \multicolumn{9}{|c|}{ C.V. $(\%)=3,9$} \\
\hline \multirow{5}{*}{ Rúcula Gigante } & 6 & 714 a & 782 a & 832 a & $834 \mathrm{a}$ & $840 \mathrm{a}$ & $842 \mathrm{a}$ & $854 \mathrm{a}$ & 903 a \\
\hline & 7 & $1198 \mathrm{~b}$ & $1298 \mathrm{~b}$ & $1353 \mathrm{~b}$ & $1369 \mathrm{~b}$ & $1372 \mathrm{~b}$ & $1378 \mathrm{~b}$ & $1386 \mathrm{~b}$ & $1389 \mathrm{~b}$ \\
\hline & 8 & $1556 \mathrm{c}$ & $1629 \mathrm{c}$ & $1689 \mathrm{c}$ & $1706 \mathrm{c}$ & $1757 \mathrm{c}$ & $1761 \mathrm{c}$ & $1762 \mathrm{c}$ & $1767 \mathrm{c}$ \\
\hline & 9 & $811 \mathrm{ab}$ & $907 \mathrm{ab}$ & $995 \mathrm{ab}$ & $1018 a b$ & $1032 a b$ & $1033 a b$ & $1040 \mathrm{ab}$ & $1055 \mathrm{ab}$ \\
\hline & 10 & $624 \mathrm{a}$ & $714 \mathrm{a}$ & $776 \mathrm{a}$ & $830 \mathrm{a}$ & $833 \mathrm{a}$ & $834 \mathrm{a}$ & $884 \mathrm{a}$ & $914 \mathrm{a}$ \\
\hline
\end{tabular}

* Letras iguais dentro de cada coluna não diferem entre si pelo teste de Tukey, a $5 \%$ de probabilidade. 
TABELA 3. Dados médios obtidos para lixiviação de potássio $(\mu \mathrm{g} \mathrm{K} / \mathrm{g})$, utilizando as combinações 100 sementes/50 mL e 100 sementes $/ 75 \mathrm{~mL}$, a $25^{\circ} \mathrm{C}$, de cinco lotes de sementes dos cultivares Rúcula Cultivada e Rúcula Gigante.

\begin{tabular}{|c|c|c|c|c|c|c|c|c|c|}
\hline \multirow{2}{*}{ Cultivares } & \multirow{2}{*}{ Lotes } & \multicolumn{8}{|c|}{ Períodos de embebição } \\
\hline & & 0,5 & 1 & 1,5 & 2 & 2,5 & 3 & 4 & 5 \\
\hline & \multicolumn{9}{|c|}{100 sementes $/ 50 \mathrm{~mL} / 25^{\circ} \mathrm{C}$} \\
\hline \multirow{5}{*}{$\begin{array}{l}\text { Rúcula } \\
\text { Cultivada }\end{array}$} & 1 & $526 \mathrm{a}$ & $652 \mathrm{a}$ & $727 \mathrm{a}$ & $775 \mathrm{a}$ & $830 \mathrm{a}$ & $864 \mathrm{a}$ & $913 \mathrm{a}$ & $916 \mathrm{a}$ \\
\hline & 2 & $715 \mathrm{~b}$ & $925 \mathrm{~b}$ & $985 \mathrm{~b}$ & $1056 \mathrm{~b}$ & $1081 \mathrm{~b}$ & $1162 \mathrm{~b}$ & $1205 \mathrm{~b}$ & $1278 \mathrm{~b}$ \\
\hline & 3 & $502 \mathrm{a}$ & $622 \mathrm{a}$ & $696 \mathrm{a}$ & $732 \mathrm{a}$ & $775 \mathrm{a}$ & $797 \mathrm{a}$ & $859 \mathrm{a}$ & $911 \mathrm{a}$ \\
\hline & 4 & $778 \mathrm{~b}$ & $1060 \mathrm{~b}$ & $1125 \mathrm{~b}$ & $1188 \mathrm{~b}$ & $1206 \mathrm{~b}$ & $1240 \mathrm{~b}$ & $1288 \mathrm{~b}$ & $1329 \mathrm{~b}$ \\
\hline & 5 & 499 a & $629 \mathrm{a}$ & $652 \mathrm{a}$ & $719 \mathrm{a}$ & $765 \mathrm{a}$ & $805 \mathrm{a}$ & $851 \mathrm{a}$ & $912 \mathrm{a}$ \\
\hline & \multicolumn{9}{|c|}{ C.V. $(\%)=4,0$} \\
\hline \multirow{7}{*}{ Rúcula Gigante } & 6 & $796 \mathrm{a}$ & 859 a & $862 \mathrm{a}$ & $894 \mathrm{a}$ & $919 \mathrm{a}$ & $944 \mathrm{a}$ & $952 \mathrm{a}$ & $1002 \mathrm{a}$ \\
\hline & 7 & $685 \mathrm{a}$ & $763 \mathrm{a}$ & $827 \mathrm{a}$ & $837 \mathrm{a}$ & $854 \mathrm{a}$ & 899 a & 939 a & $976 \mathrm{a}$ \\
\hline & 8 & $1398 \mathrm{~b}$ & $1496 \mathrm{~b}$ & $1510 \mathrm{~b}$ & $1512 \mathrm{~b}$ & $1525 \mathrm{~b}$ & $1535 \mathrm{~b}$ & $1568 \mathrm{~b}$ & $1584 \mathrm{~b}$ \\
\hline & 9 & $1259 \mathrm{~b}$ & $1338 \mathrm{~b}$ & $1339 \mathrm{~b}$ & $1341 \mathrm{~b}$ & $1353 \mathrm{~b}$ & $1364 \mathrm{~b}$ & $1374 \mathrm{~b}$ & $1405 \mathrm{~b}$ \\
\hline & 10 & $750 \mathrm{a}$ & $842 \mathrm{a}$ & $924 \mathrm{a}$ & $930 \mathrm{a}$ & $968 \mathrm{a}$ & $1003 \mathrm{a}$ & $1057 \mathrm{a}$ & $1101 \mathrm{a}$ \\
\hline & \multicolumn{9}{|c|}{ C.V. $(\%)=3,9$} \\
\hline & \multicolumn{9}{|c|}{100 sementes $/ 75 \mathrm{~mL} / 25^{\circ} \mathrm{C}$} \\
\hline \multirow{5}{*}{$\begin{array}{l}\text { Rúcula } \\
\text { Cultivada }\end{array}$} & 1 & $493 \mathrm{a}$ & $622 \mathrm{a}$ & $692 \mathrm{a}$ & 736 a & 805 a & $832 \mathrm{a}$ & 849 a & $887 \mathrm{a}$ \\
\hline & 2 & $684 \mathrm{~b}$ & $881 \mathrm{~b}$ & $951 \mathrm{~b}$ & $1025 \mathrm{~b}$ & $1057 \mathrm{~b}$ & $1128 \mathrm{~b}$ & $1168 \mathrm{~b}$ & $1243 \mathrm{~b}$ \\
\hline & 3 & $468 \mathrm{a}$ & $590 \mathrm{a}$ & $665 \mathrm{a}$ & $707 \mathrm{a}$ & $740 \mathrm{a}$ & $765 \mathrm{a}$ & $824 \mathrm{a}$ & $881 \mathrm{a}$ \\
\hline & 4 & $749 \mathrm{~b}$ & $1042 \mathrm{~b}$ & $1082 \mathrm{~b}$ & $1142 \mathrm{~b}$ & $1174 \mathrm{~b}$ & $1210 \mathrm{~b}$ & $1254 \mathrm{~b}$ & $1297 \mathrm{~b}$ \\
\hline & 5 & $465 \mathrm{a}$ & $599 \mathrm{a}$ & $626 \mathrm{a}$ & $684 \mathrm{a}$ & $734 \mathrm{a}$ & $772 \mathrm{a}$ & $819 \mathrm{a}$ & $887 \mathrm{a}$ \\
\hline & \multicolumn{9}{|c|}{ C.V. $(\%)=4,1$} \\
\hline \multirow{5}{*}{ Rúcula Gigante } & 6 & $765 \mathrm{a}$ & $822 \mathrm{a}$ & $823 \mathrm{a}$ & $866 \mathrm{a}$ & $883 \mathrm{a}$ & $901 \mathrm{a}$ & $911 \mathrm{a}$ & 979 a \\
\hline & 7 & $647 \mathrm{a}$ & $727 \mathrm{a}$ & $790 \mathrm{a}$ & $804 \mathrm{a}$ & $824 \mathrm{a}$ & $852 \mathrm{a}$ & 906 a & $932 \mathrm{a}$ \\
\hline & 8 & $1353 \mathrm{~b}$ & $1455 \mathrm{~b}$ & $1484 \mathrm{~b}$ & $1489 \mathrm{~b}$ & $1492 \mathrm{~b}$ & $1496 \mathrm{~b}$ & $1532 b$ & $1555 \mathrm{~b}$ \\
\hline & 9 & $1226 \mathrm{~b}$ & $1305 \mathrm{~b}$ & $1315 \mathrm{~b}$ & $1316 \mathrm{~b}$ & $1321 \mathrm{~b}$ & $1324 \mathrm{~b}$ & $1345 \mathrm{~b}$ & $1374 \mathrm{~b}$ \\
\hline & 10 & $729 \mathrm{a}$ & $802 \mathrm{a}$ & $897 \mathrm{a}$ & $901 \mathrm{a}$ & $935 \mathrm{a}$ & $975 \mathrm{a}$ & 1029 a & $1077 \mathrm{a}$ \\
\hline
\end{tabular}

* Letras iguais dentro de cada coluna não diferem entre si pelo teste de Tukey, a 5\% de probabilidade. 
TABELA 4. Dados médios obtidos para lixiviação de potássio ( $\mu \mathrm{g} \mathrm{K} / \mathrm{g}$ ), utilizando as combinações 50 sementes $/ 50 \mathrm{~mL}$ e 50 sementes/75 mL, a $30{ }^{\circ} \mathrm{C}$, de cinco lotes de sementes dos cultivares Rúcula Cultivada e Rúcula Gigante.

\begin{tabular}{|c|c|c|c|c|c|c|c|c|c|}
\hline \multirow{2}{*}{ Cultivares } & \multirow{2}{*}{ Lotes } & \multicolumn{8}{|c|}{ Períodos de embebição } \\
\hline & & 0,5 & 1 & 1,5 & 2 & 2,5 & 3 & 4 & 5 \\
\hline & \multicolumn{9}{|c|}{50 sementes $/ 50 \mathrm{~mL} / 30{ }^{\circ} \mathrm{C}$} \\
\hline \multirow{5}{*}{$\begin{array}{l}\text { Rúcula } \\
\text { Cultivada }\end{array}$} & 1 & $515 \mathrm{a}$ & 707 a & 792 a & 873 a & 907 a & 974 a & $1041 \mathrm{a}$ & $1176 \mathrm{a}$ \\
\hline & 2 & $891 \mathrm{~b}$ & $982 \mathrm{~b}$ & $1105 \mathrm{~b}$ & $1185 \mathrm{~b}$ & $1219 \mathrm{~b}$ & $1332 \mathrm{~b}$ & $1366 \mathrm{~b}$ & $1478 \mathrm{~b}$ \\
\hline & 3 & $486 \mathrm{a}$ & $639 \mathrm{a}$ & $736 \mathrm{a}$ & $805 \mathrm{a}$ & $836 \mathrm{a}$ & $903 \mathrm{a}$ & 979 a & $1065 \mathrm{a}$ \\
\hline & 4 & $932 \mathrm{~b}$ & $1082 \mathrm{~b}$ & $1164 b$ & $1416 \mathrm{c}$ & $1545 \mathrm{c}$ & $1610 \mathrm{c}$ & $1651 \mathrm{c}$ & $1767 \mathrm{c}$ \\
\hline & 5 & $513 \mathrm{a}$ & $690 \mathrm{a}$ & $761 \mathrm{a}$ & 824 a & $903 \mathrm{a}$ & 947 a & $1035 \mathrm{a}$ & 1109 a \\
\hline & \multicolumn{9}{|c|}{ C.V. $(\%)=4,3$} \\
\hline \multirow{7}{*}{ Rúcula Gigante } & 6 & $846 \mathrm{a}$ & $926 \mathrm{a}$ & $935 \mathrm{a}$ & $981 \mathrm{a}$ & $982 \mathrm{a}$ & $994 \mathrm{a}$ & $1124 \mathrm{a}$ & $1168 \mathrm{a}$ \\
\hline & 7 & $1339 \mathrm{~b}$ & $1341 \mathrm{~b}$ & $1379 b$ & $1395 \mathrm{~b}$ & $1398 \mathrm{~b}$ & $1406 \mathrm{~b}$ & $1412 \mathrm{~b}$ & $1449 \mathrm{~b}$ \\
\hline & 8 & $1515 \mathrm{~b}$ & $1608 \mathrm{~b}$ & $1613 \mathrm{~b}$ & $1621 \mathrm{c}$ & $1635 \mathrm{c}$ & $1651 \mathrm{c}$ & $1729 \mathrm{c}$ & $1746 \mathrm{c}$ \\
\hline & 9 & $1483 b$ & $1582 b$ & $1625 \mathrm{~b}$ & $1639 \mathrm{c}$ & $1664 \mathrm{c}$ & $1739 \mathrm{c}$ & $1744 \mathrm{c}$ & $1755 \mathrm{c}$ \\
\hline & 10 & 828 a & $916 \mathrm{a}$ & $978 \mathrm{a}$ & $1037 \mathrm{a}$ & $1079 \mathrm{a}$ & $1092 \mathrm{a}$ & 1148 a & $1162 \mathrm{a}$ \\
\hline & \multicolumn{9}{|c|}{ C.V. $(\%)=4,5$} \\
\hline & \multicolumn{9}{|c|}{50 sementes $/ 75 \mathrm{~mL} / 30^{\circ} \mathrm{C}$} \\
\hline \multirow{5}{*}{$\begin{array}{l}\text { Rúcula } \\
\text { Cultivada }\end{array}$} & 1 & $451 \mathrm{a}$ & $642 \mathrm{a}$ & $734 \mathrm{a}$ & $814 \mathrm{a}$ & $841 \mathrm{a}$ & $912 \mathrm{a}$ & $984 \mathrm{a}$ & $1116 \mathrm{a}$ \\
\hline & 2 & $839 \mathrm{~b}$ & $921 \mathrm{~b}$ & $1046 \mathrm{~b}$ & $1122 b$ & $1153 \mathrm{~b}$ & $1274 \mathrm{~b}$ & $1309 \mathrm{~b}$ & $1411 b$ \\
\hline & 3 & $425 \mathrm{a}$ & 578 a & $671 \mathrm{a}$ & $750 \mathrm{a}$ & $775 \mathrm{a}$ & $845 \mathrm{a}$ & $915 \mathrm{a}$ & $1005 \mathrm{a}$ \\
\hline & 4 & $888 \mathrm{~b}$ & $1036 \mathrm{~b}$ & $1110 \mathrm{~b}$ & $1155 \mathrm{~b}$ & $1183 \mathrm{~b}$ & $1257 \mathrm{~b}$ & $1292 \mathrm{~b}$ & $1409 \mathrm{~b}$ \\
\hline & 5 & $456 \mathrm{a}$ & $638 \mathrm{a}$ & $704 \mathrm{a}$ & $764 \mathrm{a}$ & $848 \mathrm{a}$ & 889 a & $980 \mathrm{a}$ & $1044 \mathrm{a}$ \\
\hline & \multicolumn{9}{|c|}{ C.V. $(\%)=4,2$} \\
\hline \multirow{5}{*}{ Rúcula Gigante } & 6 & $782 \mathrm{a}$ & $885 \mathrm{a}$ & $887 \mathrm{a}$ & $927 \mathrm{a}$ & $929 \mathrm{a}$ & $941 \mathrm{a}$ & $1061 \mathrm{a}$ & $1111 \mathrm{a}$ \\
\hline & 7 & $1276 \mathrm{~b}$ & $1287 \mathrm{~b}$ & $1319 b$ & $1332 \mathrm{~b}$ & $1342 \mathrm{~b}$ & $1345 \mathrm{~b}$ & $1354 \mathrm{~b}$ & $1395 \mathrm{~b}$ \\
\hline & 8 & $1453 \mathrm{c}$ & $1548 \mathrm{c}$ & $1553 \mathrm{c}$ & $1559 \mathrm{c}$ & $1585 \mathrm{c}$ & $1596 \mathrm{c}$ & $1667 \mathrm{c}$ & $1682 \mathrm{c}$ \\
\hline & 9 & $1220 \mathrm{~b}$ & $1226 \mathrm{~b}$ & $1269 b$ & $1285 \mathrm{~b}$ & $1310 \mathrm{~b}$ & $1374 \mathrm{~b}$ & $1384 \mathrm{~b}$ & $1393 \mathrm{~b}$ \\
\hline & 10 & 766 a & $853 \mathrm{a}$ & $921 \mathrm{a}$ & $983 \mathrm{a}$ & $1022 \mathrm{a}$ & $1037 \mathrm{a}$ & $1090 \mathrm{a}$ & $1105 \mathrm{a}$ \\
\hline
\end{tabular}

* Letras iguais dentro de cada coluna não diferem entre si pelo teste de Tukey, a 5\% de probabilidade. 
TABELA 5. Dados médios obtidos para lixiviação de potássio $(\mu \mathrm{g} \mathrm{K} / \mathrm{g})$, utilizando as combinações 100 sementes/50 mL e 100 sementes/75 mL, a $30{ }^{\circ} \mathrm{C}$, de cinco lotes de sementes dos cultivares Rúcula Cultivada e Rúcula Gigante.

\begin{tabular}{|c|c|c|c|c|c|c|c|c|c|}
\hline \multirow{2}{*}{ Cultivares } & \multirow{2}{*}{ Lotes } & \multicolumn{8}{|c|}{ Períodos de embebição } \\
\hline & & 0,5 & 1 & 1,5 & 2 & 2,5 & 3 & 4 & 5 \\
\hline & \multicolumn{9}{|c|}{$100 \mathrm{sEmentes} / 50 \mathrm{~mL} / 30^{\circ} \mathrm{C}$} \\
\hline \multirow{5}{*}{$\begin{array}{l}\text { Rúcula } \\
\text { Cultivada }\end{array}$} & 1 & $551 \mathrm{a}$ & $674 \mathrm{a}$ & $740 \mathrm{a}$ & $817 \mathrm{a}$ & $872 \mathrm{a}$ & $914 \mathrm{a}$ & $997 \mathrm{a}$ & $1075 \mathrm{a}$ \\
\hline & 2 & $546 \mathrm{a}$ & $685 \mathrm{a}$ & $777 \mathrm{a}$ & $864 \mathrm{a}$ & $931 \mathrm{a}$ & $1145 \mathrm{~b}$ & $1192 \mathrm{~b}$ & $1250 \mathrm{~b}$ \\
\hline & 3 & $973 \mathrm{c}$ & $1103 \mathrm{c}$ & $1176 \mathrm{c}$ & $1220 \mathrm{c}$ & $1270 \mathrm{c}$ & $1319 \mathrm{c}$ & $1398 \mathrm{c}$ & $1441 \mathrm{c}$ \\
\hline & 4 & $827 \mathrm{bc}$ & $944 \mathrm{bc}$ & $1012 \mathrm{bc}$ & $1105 \mathrm{bc}$ & $1165 \mathrm{bc}$ & $1195 \mathrm{bc}$ & $1272 \mathrm{bc}$ & $1312 \mathrm{bc}$ \\
\hline & 5 & 596 a & $678 \mathrm{a}$ & $744 \mathrm{a}$ & $796 \mathrm{a}$ & $849 \mathrm{a}$ & $888 \mathrm{a}$ & $964 \mathrm{a}$ & $1053 \mathrm{a}$ \\
\hline & \multicolumn{9}{|c|}{ C.V. $(\%)=4,1$} \\
\hline \multirow{7}{*}{ Rúcula Gigante } & 6 & $812 \mathrm{a}$ & $875 \mathrm{a}$ & $1036 \mathrm{a}$ & $1037 \mathrm{a}$ & $1079 \mathrm{a}$ & $1095 \mathrm{a}$ & $1156 \mathrm{a}$ & $1159 \mathrm{a}$ \\
\hline & 7 & $1263 \mathrm{~b}$ & $1385 \mathrm{~b}$ & $1421 \mathrm{~b}$ & $1425 \mathrm{~b}$ & $1435 \mathrm{~b}$ & $1439 \mathrm{~b}$ & $1447 \mathrm{~b}$ & $1449 \mathrm{~b}$ \\
\hline & 8 & $794 \mathrm{a}$ & $919 \mathrm{a}$ & $967 \mathrm{a}$ & $976 \mathrm{a}$ & $979 \mathrm{a}$ & $995 \mathrm{a}$ & $996 \mathrm{a}$ & 997 a \\
\hline & 9 & $1407 \mathrm{c}$ & $1518 \mathrm{c}$ & $1565 \mathrm{~b}$ & $1568 \mathrm{~b}$ & $1572 b$ & $1576 \mathrm{~b}$ & $1579 \mathrm{~b}$ & $1585 \mathrm{~b}$ \\
\hline & 10 & $745 \mathrm{a}$ & $864 \mathrm{a}$ & $932 \mathrm{a}$ & $945 \mathrm{a}$ & $975 \mathrm{a}$ & $994 \mathrm{a}$ & $1031 \mathrm{a}$ & $1037 \mathrm{a}$ \\
\hline & \multicolumn{9}{|c|}{ C.V. $(\%)=4,7$} \\
\hline & \multicolumn{9}{|c|}{100 sementes $/ 75 \mathrm{~mL} / 30^{\circ} \mathrm{C}$} \\
\hline \multirow{5}{*}{$\begin{array}{c}\text { Rúcula } \\
\text { Cultivada }\end{array}$} & 1 & $525 \mathrm{a}$ & $633 \mathrm{a}$ & $708 \mathrm{a}$ & $772 \mathrm{a}$ & $833 \mathrm{a}$ & $878 \mathrm{a}$ & $952 \mathrm{a}$ & $1036 \mathrm{a}$ \\
\hline & 2 & $507 \mathrm{a}$ & $642 \mathrm{a}$ & $736 \mathrm{a}$ & $821 \mathrm{a}$ & $892 \mathrm{a}$ & $1109 \mathrm{~b}$ & $1151 \mathrm{~b}$ & $1215 \mathrm{~b}$ \\
\hline & 3 & $935 c$ & $1061 \mathrm{~b}$ & $1137 \mathrm{~b}$ & $1188 \mathrm{~b}$ & $1248 \mathrm{~b}$ & $1275 \mathrm{c}$ & $1355 \mathrm{c}$ & $1414 \mathrm{c}$ \\
\hline & 4 & $789 \mathrm{~b}$ & $908 \mathrm{~b}$ & $977 \mathrm{~b}$ & $1063 \mathrm{~b}$ & $1129 \mathrm{~b}$ & $1152 \mathrm{bc}$ & $1234 \mathrm{bc}$ & $1278 \mathrm{bc}$ \\
\hline & 5 & $562 \mathrm{a}$ & $637 \mathrm{a}$ & $701 \mathrm{a}$ & $751 \mathrm{a}$ & $810 \mathrm{a}$ & $841 \mathrm{a}$ & $927 \mathrm{a}$ & $1018 \mathrm{a}$ \\
\hline & \multicolumn{9}{|c|}{ C.V. $(\%)=4,5$} \\
\hline \multirow{6}{*}{ Rúcula Gigante } & 6 & $774 \mathrm{a}$ & $871 \mathrm{a}$ & $990 \mathrm{a}$ & $995 \mathrm{a}$ & $1035 \mathrm{a}$ & $1054 \mathrm{a}$ & $1112 \mathrm{a}$ & $1114 \mathrm{a}$ \\
\hline & 7 & $1228 \mathrm{~b}$ & $1345 b$ & $1381 \mathrm{~b}$ & $1384 \mathrm{~b}$ & $1397 \mathrm{~b}$ & $1403 \mathrm{~b}$ & $1406 \mathrm{~b}$ & $1409 \mathrm{~b}$ \\
\hline & 8 & $760 \mathrm{a}$ & $878 \mathrm{a}$ & $925 \mathrm{a}$ & $933 \mathrm{a}$ & $938 \mathrm{a}$ & $950 \mathrm{a}$ & $955 \mathrm{a}$ & $965 \mathrm{a}$ \\
\hline & 9 & $1372 \mathrm{c}$ & $1474 \mathrm{c}$ & $1537 \mathrm{c}$ & $1538 \mathrm{c}$ & $1541 \mathrm{c}$ & $1545 \mathrm{c}$ & $1549 \mathrm{c}$ & $1551 \mathrm{c}$ \\
\hline & 10 & $704 \mathrm{a}$ & $826 \mathrm{a}$ & $896 \mathrm{a}$ & $906 \mathrm{a}$ & $939 \mathrm{a}$ & $958 \mathrm{a}$ & $1013 \mathrm{a}$ & $1034 \mathrm{a}$ \\
\hline & \multicolumn{9}{|c|}{ C.V. $(\%)=3,9$} \\
\hline
\end{tabular}

- Letras iguais dentro de cada coluna não diferem entre si pelo teste de Tukey, a 5\% de probabilidade.

Observou-se que a $25{ }^{\circ} \mathrm{C}$, utilizando 50 ou 100 sementes, e variando o volume de água ( 50 ou $75 \mathrm{~mL})$, não houve a separação dos lotes de níveis de vigor intermediários (Tabelas 2 e 3), prejudicando a recomendação deste teste, nessas condições. O mesmo ocorreu a $30^{\circ} \mathrm{C}$ (Tabela 5) utilizando-se 100 sementes.

A combinação 50 sementes $/ 50 \mathrm{~mL} / 30{ }^{\circ} \mathrm{C}$ (Tabela 4), apresentou melhor distinção dos lotes, para ambos os cultivares, a partir de 2 horas de embebição das sementes. Dessa forma, o lote 4 da Rúcula Cultivada foi indicado como o de menor potencial fisiológico, os lotes 1,3 e 5 como os de melhor qualidade, e o lote 2 como intermediário. Para a Rúcula Gigante, os lotes 6 e 10 foram apontados como os de maior potencial fisiológico, os lotes 8 e 9 como os de pior desempenho, e o lote 7 como intermediário. Esses resultados se mostraram coerentes com o teste de primeira contagem de germinação e emergência em casa de vegetação (Tabela 1 ).

A análise da correlação entre os resultados da avaliação da qualidade fisiológica das sementes e de emergência das plântulas em campo (Tabela 6), indicou que os testes de germinação e primeira contagem de germinação 
correlacionaram-se positiva e significativamente $(\mathrm{P}<0,05)$ com a emergência das plântulas em campo, confirmando resultados anteriores obtidos por Lima (1993) e Andrade et al. (1995) avaliando lotes de sementes de cebola e cenoura, respectivamente.

TABELA 6. Coeficiente de correlação simples (r) estimados entre os testes de avaliação da qualidade fisiológica das sementes e emergência em casa de vegetação, em dois cultivares e cinco lotes de cada cultivar de sementes de rúcula.

\begin{tabular}{lcccc}
\hline Cultivares & Testes & PCG & LP & EMERG \\
\hline \multirow{4}{*}{ Rúcula Cultivada } & G & $0,983^{*}$ & $-0,897^{*}$ & $0,955^{*}$ \\
& LP & - & $-0,903^{*}$ & $0,942^{*}$ \\
& G & $0,927^{*}$ & $-0,915^{*}$ & $0,932^{*}$ \\
Rúcula Gigante & PCG & - & $-0,899^{*}$ & $0,916^{*}$ \\
& LP & - & - & $0,873^{*}$ \\
\hline
\end{tabular}

$\mathrm{G}=$ teste de germinação

$\mathrm{PCG}=$ primeira contagem de germinação

$\mathrm{LP}=$ lixiviação de potássio

EMERG = emergência de plântulas em casa de vegetação

* $\mathrm{r}$ significativo a $5 \%$ de probabilidade.

Os resultados da correlação significativa $(\mathrm{P}<0,05)$ e negativa, entre o teste de lixiviação de potássio e a emergência das plântulas em casa de vegetação são similares aos encontrados por Andrade et al. (1995), Torres et al. (1999) e Ávila et al. (2005), em sementes de cenoura, maxixe e canola, respectivamente, porém com o teste de condutividade elétrica, cujo princípio também se baseia na integridade das membranas celulares.

De forma geral, para ambos os cultivares, verificou-se que houve significativa lixiviação de potássio logo no período inicial de embebição, ou seja, 30 minutos, continuando a aumentar com menos intensidade até o período de 5 horas de embebição, fato que também foi confirmado por Torres (2002), em sementes de melão. Dias et al (1998), em sementes de feijão-de-vagem verificaram leituras mais elevadas de lixiviação de potássio aos 120 minutos de embebição das sementes. Este fato, provavelmente se deu pelo próprio processo de embebição, onde a absorção de água em sementes com grau de umidade inicial baixo (em torno de $5,5 \%$ - Tabela 1) apresenta velocidade favorecida pelo alto gradiente de potencial hídrico entre o interior da semente e a água circundante, além de seu tamanho relativamente pequeno, comprovando, nessas condições, ser um teste bastante promissor na separação de diferentes níveis de vigor em sementes de rúcula.

\section{CONCLUSÕES}

Diante do exposto, concluiu-se que o teste de lixiviação de potássio é eficiente na separação dos lotes de rúcula com relação ao potencial fisiológico, sendo que a condição mais adequada para a realização deste teste é a utilização de 50 sementes em $50 \mathrm{~mL}$ de água, por 2 horas, a $30^{\circ} \mathrm{C}$.

\section{REFERÊNCIAS}

AVILA, M.R.; BRACCINI, A.L.; SCAPIM, C.A.; MARTORELLI, D.T.; ALBRECHT, L.P. Testes de laboratório em sementes de canola e a correlação com a emergência das plântulas em campo. Revista Brasileira de Sementes, v.27, n.1, p.62-70, 2005.

ANDRADE, R.N.; SANTOS, D.S.B.; SANTOS FILHO, B.G.; MELLO, V.D.C. Correlação entre testes de vigor em sementes de cenoura armazenadas por diferentes períodos. Pesquisa Agropecuária Gaúcha, v.1, n.2, p.153-162, 1995.

BRASIL. Ministério da Agricultura e Reforma Agrária. Secretaria Nacional de Defesa Agropecuária. Departamento Nacional de Defesa Vegetal. Coordenação de Laboratório Vegetal. Regras para análise de sementes. Brasília, DF, 1992. 365p

CUSTODIO, C.C.; MARCOS FILHO, J. Potassium leachate test for the evaluation of soybean seed physiological quality. Seed Science and Technology, v.25, n.3, p.549-564, 1997.

DIAS, D.C.F.S.; MARCOS FILHO, J. Testes de vigor baseados na permeabilidade das membranas celulares: I. Condutividade elétrica. Informativo ABRATES, v.5, n.1, p.26-36, 1995.

DIAS, D.C.F.S.; VIEIRA, A.N.; BHERING, M.C. Condutividade elétrica e lixiviação de potássio para avaliação do vigor de sementes de hortaliças: feijão-de-vagem e quiabo. Revista Brasileira de Sementes, v.20, n.2, p.408413, 1998.

HALLOIN, J.M. Solute loss deteriorated cotton seed: relationship between deterioration, seed moisture and solute loss. Crop Science, v.15, n.1, p.11-15, 1975.

HÖFS, A.; SCHUCH, L.O.B.; PESKE, S.T.; BARROS, A.C.S.A. Emergência e crescimento de plântulas de arroz em resposta à qualidade fisiológica de sementes. Revista 
Brasileira de Sementes, v.26, n.1, p.92-97, 2004.

KOLCHINSKI, E.M. ; SCHUCH, L.O.B. ; PESKE, S.T. . Crescimento inicial de soja em função do vigor de sementes. Revista Brasileira de Agrociência, v.12, p.163-166, 2006.

LARSEN, S.U., POVLSEN, F.V., ERIKSEN, E.N., PEDERSEN, H.C. The influence of seed vigor on field performance and the evaluation of the applicability of the controlled deterioration vigor test in oil seed rape (Brassica napus) and pea (Pisum sativum). Seed Science and Technology, v.26, n.3, p.627-41, 1998.

LIMA, D. Avaliação da viabilidade e vigor de sementes de cebola (Allium cepa L.). Pelotas. 1993. 61f. Dissertação (Mestrado em Ciência e Tecnologia de Sementes) Faculdade de Agronomia Eliseu Maciel, Universidade Federal de Pelotas, Pelotas, 1993.

LOTT, J.N.A.; CAVDEK, V.; CARSON, J. Leakage of K, $\mathrm{Mg}, \mathrm{Cl}, \mathrm{Ca}$ and $\mathrm{Mn}$ from imbibing seeds, grains and isolated seed parts. Seed Science Research, v.1, n.4, p.229-233, 1991.

MARCOS FILHO, J.; AMORIM, H.V.; SILVAROLLA, M.B.; PESCARIN, H.M.C. Relações entre germinação, vigor e permeabilidade das membranas celulares durante a maturação de sementes de soja. In: SEMINÁRIO NACIONAL DA PESQUISA DE SOJA, 2., 1981, Brasília, DF. Anais... Londrina: EMBRAPA/ CNPSo, 1982. v.1. p.676-688.

MARCOS FILHO, J. Testes de vigor: importância e utilização. In: KRZYZANOWSKI, F.C.; VIEIRA, R.D.; FRANÇA NETO, J.B. (Ed.). Vigor de sementes: conceitos e testes. Londrina: ABRATES, 1999. p.1-21.

McKERSIE, B.D.; STINSON, R.H. Effect of dehydration on leakage and membrane structure in Lotus corniculatus L. seeds. Plant Physiology, v.66, n.2, p.316-320, 1980.

MIGUEL, M.V.C. Avaliação do potencial fisiológico de sementes de milho através do teste de lixiviação de potássio. 2001. 113f. Tese (Doutorado) - Escola Superior de Agricultura "Luiz de Queiroz", Universidade de São Paulo, Piracicaba, 2001.

MOSS, G.I.; MULLET, J.H. Potassium release and seed vigour in germination bean (Phaseolus vulgaris L.) seed as influenced by temperature over the previous five generations. Journal of Experimental Botany, v.33, n.137, p.1147-1160,
1982.

MULLETT, J.H.; CONSIDINE, J.A. Potassium release and uptake in germination legume seeds in relation to seed condition and germination environment. Journal of Experimental Botany, v.31, p.151-162, 1980.

PANOBIANCO, M.; MARCOS FILHO, J. Evaluation of the physiological potential of tomato seeds by germination and vigor tests. Seed Technology, v.23, n.2, p.151-161, 2001.

PIANA, Z.; TILLMANN, M.A.A.; MINAMI, K. Avaliação da qualidade fisiológica de sementes de cebola e sua relação com a produção de mudas vigorosas. Revista Brasileira de Sementes, v.17, n.2, p.149-153, 1995.

PURQUERIO, L.F.V. Crescimento, produção e qualidade de rúcula (Eruca sativa Miller) em função do nitrogênio e da densidade de plantio. 2005. 138f. Tese (Doutorado) Faculdade de Ciências Agronômicas, Universidade Estadual Paulista, Botucatu, 2005.

SIMON, E.W.; MATHAVAN, S. The time-course of leakage from imbibing seeds of different species. Seed Science and Technology, v.14, n.1, p.9-13, 1986.

SIMON, E.W.; RAJA-HARUN, R.M. Leakage during seed imbibition. Journal of Experimental Botany, v.23, n.77, p.1076-1085, 1972.

TORRES, S.B. Métodos para avaliação do potencial fisiológico de sementes de melão. 2002. 103f. Tese (Doutorado) - Escola Superior de Agricultura "Luiz de Queiroz", Universidade de São Paulo, Piracicaba, 2002.

TORRES, S.B.; SILVA, M.A.S.; CARVALHO, I.M.S.; QUEIRÓZ, M.A. Correlação entre testes de vigor em sementes de maxixe. Pesquisa Agropecuária Brasileira, v.34, n.6, p.1075-1080, 1999.

WOODSTOCK, L.W.; FURMAN, K.; LEFFLER, H.R. Relationship between weathering deterioration and germination, respiratory metabolism and mineral leaching from cotton seeds. Crop Science, v.25, n.3, p.459-466, 1985.

ZONTA, E.P.; MACHADO, A.A. Sistema de análise estatística para microcomputadores - SANEST. Pelotas: UFPel, 1984. 75p. (Registro SEI n.06606-0, categoria AO). 